\title{
THE SUBNORMAL SUBGROUP STRUCTURE OF THE INFINITE SYMPLECTIC GROUP
}

\author{
by DAVID G. ARRELL
}

(Received 10th October 1980)

\section{Introduction}

Let $R$ be a ring with identity and let $\Omega$ be a totally ordered set. Let $\Omega^{\prime}$ be a totally ordered set which is disjoint from and equipotent to $\Omega$ with ' $: \Omega \rightarrow \Omega^{\prime}$ an order preserving bijection. Define $\Omega_{1}=\Omega \cup \Omega^{\prime}$ and let $\Omega_{1}$ be totally ordered by inheriting the order from $\Omega$ and $\Omega^{\prime}$ and with $\omega<\lambda^{\prime}$, for all $\omega \in \Omega$ and $\lambda^{\prime} \in \Omega^{\prime}$. Let $M$ be the free $R$-module $R^{\left(\Omega_{1}\right)}$. We define the alternate bilinear form $\left({ }^{*},{ }^{*}\right)$ on $M$ by

$$
\begin{aligned}
& \left.\begin{array}{l}
\left(e_{\omega}, e_{\omega^{\prime}}\right)=1 \\
\left(e_{\omega^{\prime}}, e_{\omega}\right)=-1
\end{array}\right\} \text { for all } \omega \in \Omega \\
& \left.\begin{array}{r}
\left(e_{\alpha}, e_{\beta}\right)=0 \\
\left(e_{\alpha^{\prime}}, e_{\beta^{\prime}}\right)=0
\end{array}\right\} \quad \text { for all } \alpha, \beta \in \Omega, \\
& \left.\begin{array}{l}
\left(e_{\alpha^{\prime}}, e_{\beta}\right)=0 \\
\left(e_{\alpha}, e_{\beta^{\prime}}\right)=0
\end{array}\right\} \quad \text { for all } \alpha, \beta \in \Omega, \quad \alpha \neq \beta \text {. }
\end{aligned}
$$

The symplectic group $S p(\Omega, R)$ is the group of $R$-automorphisms $X$ of $M$ for which $(X(x), X(y))=(x, y)$, for all $x, y \in M$. We see that $S p(\Omega, R)$ is a subgroup of $G L\left(\Omega_{1}, R\right)$ and, since $\Omega_{1}$ is totally ordered, we can think of any $X \in S p(\dot{\Omega}, R)$ as an invertible $\Omega_{1} \times \Omega_{1}$ matrix over $R$ partitioned as

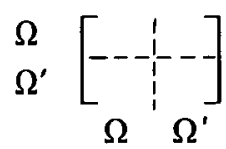

We shall call $X \in S p(\Omega, R)$ a symplectic transformation or matrix. If we let $J$ denote the $\Omega_{1} \times \Omega_{1}$ matrix

$$
\left[\begin{array}{rr}
0 & I \\
-I & 0
\end{array}\right]
$$

where $I$ is the $\Omega \times \Omega$ identity matrix then clear that $X$ is a symplectic matrix if and only if $X^{\prime} J X=J .\left(X^{\prime}\right.$ denotes the transpos $\_$matrix of $X$.) 
The structure of $S p(\Omega, R)$ has been the subject of many investigations: when $\Omega$ is finite and $R$ is a division ring, Dieudonné studied $S p(\Omega, R)$ in (4) and (5); when $\Omega$ is infinite, Spiegel in (8) examined $S p(\Omega, R)$ when $R$ is a division ring and Maxwell in (6) classified the normal subgroups of $S p(\Omega, R)$ when $R$ is a commutative ring in which 2 is a unit. We continue this investigation to give a classification of the subnormal subgroups of $\operatorname{Sp}(\Omega, R)$ when $\Omega$ is infinite and $R$ is a commutative ring with identity. The classification we shall obtain is similar to the "sandwiching" results of Bass (3) and Wilson (9) in which the congruence subgroups play a major rôle.

\section{Preliminaries}

For the rest of this paper $\Omega$ shall always denote an infinite set and all rings $R$ will have identity. Let $a \in R, \alpha, \beta \in \Omega_{1}$; we denote the $\Omega_{1} \times \Omega_{1}$ matrix whose only non-zero entry is in the $(\alpha, \beta)$ th position and is $a$ by $a e_{\alpha \beta}$. We let 1 denote the $\Omega_{1} \times \Omega_{1}$ identity matrix. Following Robertson (7), we define the $\Omega_{1} \times \Omega_{1}$ matrices $t_{\lambda \mu}(a), r_{\lambda \mu}(a), r_{\lambda \lambda}(a), s_{\lambda \mu}(a)$ and $s_{\lambda \lambda}(a)$, for $a \in R$ and $\lambda, \mu \in \Omega, \lambda \neq \mu$, by

$$
\begin{aligned}
& t_{\lambda \mu}(a)=1+a\left(e_{\lambda \mu}-e_{\mu^{\prime} \lambda^{\prime}}\right), \\
& r_{\lambda \mu}(a)=1+a\left(e_{\lambda \mu^{\prime}}+e_{\mu \lambda^{\prime}}\right), \\
& r_{\lambda \lambda}(a)=1+a e_{\lambda \lambda^{\prime}}, \\
& s_{\lambda \mu}(a)=1+a\left(e_{\lambda^{\prime} \mu}+e_{\mu^{\prime} \lambda}\right), \\
& s_{\lambda \lambda}(a)=1+a e_{\lambda^{\prime} \lambda} .
\end{aligned}
$$

We also quote from (7) the following lemma which is a list of commutator identities.

Lemma 1. Let $R$ be a commutative ring, $a, b \in R$ and $\lambda, \mu, \alpha, \beta \in \Omega$.
(i) $\left[r_{\lambda \mu}(b), s_{\alpha \beta}(a)\right]=1$,
(ii) $\left[r_{\lambda \alpha}(b), s_{\alpha \beta}(a)\right]=t_{\lambda \beta}(b a)$
$\lambda, \mu$ distinct from $\alpha, \beta$,
(iii) $\left[r_{\lambda \alpha}(b), s_{\alpha \alpha}(a)\right]=t_{\lambda \alpha}(b a) r_{\lambda \lambda}\left(b^{2} a\right)$
$\alpha, \beta, \lambda$ distinct,
(iv) $\left[r_{\lambda \lambda}(b), s_{\alpha \lambda}(a)\right]=t_{\lambda \alpha}(b a) s_{\alpha a}\left(-b a^{2}\right)$
$\lambda \neq \alpha$,
(v) $\left[s_{\lambda \mu}(a), t_{\lambda \beta}(b)\right]=s_{\mu \beta}(a b)$
$\lambda \neq \alpha$,
(vi) $\left[s_{\lambda \mu}(a), t_{\alpha \mu}(b)\right]=1$
(vii) $\left[s_{\lambda \mu}(a), t_{\lambda \mu}(b)\right]=s_{\mu \mu}(2 a b)$
$\lambda, \mu, \beta$ distinct,
(viii) $\left[s_{\lambda \lambda}(a), t_{\lambda \mu}(b)\right]=s_{\lambda \mu}(a b) s_{\mu \mu}\left(a b^{2}\right)$
$\alpha \neq \lambda, \mu$,
(ix) $\left[s_{\lambda \mu}(a), t_{\alpha \beta}(b)\right]=1$
(x) $\left[r_{\lambda \mu}(a), t_{\alpha \beta}(b)\right]=1$
(xi) $\left[r_{\lambda \mu}(a), t_{\alpha \mu}(b)\right]=r_{\alpha \lambda}(-a b)$
$\lambda \neq \mu$,
$\lambda \neq \mu$,
$\lambda, \mu$ distinct from $\alpha, \beta$,
(xii) $\left[r_{\lambda \mu}(a), t_{\lambda \beta}(b)\right]=1$
$\lambda, \mu$ distinct from $\alpha, \beta$,
(xiii) $\left[r_{\lambda \mu}(a), t_{\lambda \mu}(b)\right]=r_{\lambda \lambda}(-2 a b)$
$\lambda, \mu, \alpha$ distinct,
(xiv) $\left[r_{\lambda \lambda}(a), t_{a \lambda}(b)\right]=r_{a \lambda}(-a b) r_{a \alpha}\left(a b^{2}\right) \quad \lambda \neq \mu$,
$\beta \neq \lambda, \mu$,
$\lambda \neq \mu$,
(xiv) $\left[r_{\lambda \lambda}(a), t_{\alpha \lambda}(b)\right]=r_{\alpha \lambda}(-a b) r_{\alpha \alpha}\left(a b^{2}\right) \quad \lambda \neq \alpha$.

We define $\operatorname{ESp}(\Omega, R)$ to be the subgroup of $S p(\Omega, R)$ generated by $\left\{r_{\lambda \mu}(a), s_{\lambda \mu}(a): \lambda, \mu \in \Omega\right.$, $a \in R\}$. For any two-sided ideal $\mathfrak{p}$ of $R, \operatorname{ESp}(\Omega, \mathfrak{p})$ will denote the normal closure of 
$\left\{r_{\lambda \mu}(a), s_{\lambda \mu}(a): \lambda, \mu \in \Omega, a \in \mathfrak{p}\right\}$ in $\operatorname{ESp}(\Omega, R), S p(\Omega, p)$ will denote the kernel of the group homomorphism $S p(\Omega, R) \rightarrow S p(\Omega, R / p)$ induced by the projection $R \rightarrow R / p$ and $S p^{\prime}(\Omega, p)$ will denote the inverse image of the centre of $S p(\Omega, R / p)$. Clearly

$$
\operatorname{ESp}(\Omega, \mathfrak{p}) \leqq S p(\Omega, \mathfrak{p}) \leqq S p^{\prime}(\Omega, \mathfrak{p})
$$

It is easy to show that the groups $\operatorname{ESp}(\Omega, R)$ and $\operatorname{ESp}(\Omega, \mathfrak{p})$ coincide with $\operatorname{ESp}(R)$ and $E S p(p)$ of Maxwell (6). It follows from (6) that when $R$ is commutative $\operatorname{ESp}(\Omega, R)$ and $\operatorname{ESp}(\Omega, \mathfrak{p})$ are normal subgroups of $S p(\Omega, R)$. However, the proofs of 2.2-2.5 of (6) do not depend upon the commutativity of $R$ and thus we may state

Lemma 2. For any two-sided ideal $\mathfrak{p}$ of a ring $R, \operatorname{ESp}(\Omega, R)$ and $\operatorname{ESp}(\Omega, p)$ are normal subgroups of $\operatorname{Sp}(\Omega, R)$ and $\operatorname{ESp}(\Omega, \mathfrak{p})=\left[\operatorname{ESp}(\Omega, R), S p^{\prime}(\Omega, \mathfrak{p})\right]$.

Simple matrix arguments show that the centre of $S p(\Omega, R)$ comprises all matrices of the form

$$
\left[\begin{array}{cc}
r I & 0 \\
0 & r I
\end{array}\right],
$$

for all central units $r$ of $R$ with $r^{2}=1$. Since all matrices in $\operatorname{ESp}(\Omega, R)$ differ from the identity matrix in only finitely many rows, we see that $\operatorname{ESp}(\Omega, R)$ has trivial centre. From these remarks we are able to deduce

Lemma 3. Let $R$ be a commutative ring. If $H$ is a subgroup of $S p(\Omega, R)$ that is normalised by $\operatorname{ESp}(\Omega, R)$ then the following assertions are equivalent.

(i) $H \leqq Z(S p(\Omega, R))$.

(ii) $[H, \operatorname{ESp}(\Omega, R)]=1$.

(iii) $H \cap E S p(\Omega, R)=1$.

(For any group $G$ we use $Z(G)$ to denote the centre of $G$.)

For any symplectic matrix $X$ we let $J(X)$ denote the two-sided ideal generated by the matrix entries $X_{\alpha \beta}, X_{\alpha z}-X_{\beta \beta}$, for all $\alpha, \beta \in \Omega_{1}, \alpha \neq \beta$ (c.f. (9)). We shall call $J(X)$ the level of $X$ and the entries $X_{\alpha \beta}$ and $X_{\alpha \alpha}-X_{\beta \beta}$ the generators of $J(X)$. For any subgroup $H$ of $\operatorname{Sp}(\Omega, R)$ we denote by $J(H)$ the ideal which is the sum of the ideals $J(X)$, for all $X \in H$, and by $K(H)$ the ideal which is the sum of the ideals $J(X)$, for all $X \in H \cap E S p(\Omega, R)$, (c.f. (2)). For any commutative ring $R$ and any $X \in S p(\Omega, R)$ it is clear that $J(X)$ is the least ideal $p$ of $R$ such that $X \in S p^{\prime}(\Omega, p)$ and that $X \in Z(S p(\Omega, R))$ if and only if $J(X)=0$.

We now complete this section by recalling some general group theoretic definitions. We say that a subgroup $H$ of a group $G$ is a subnormal subgroup of $G$ if there exists a normal series of subgroups

$$
H=H_{d} \triangleleft H_{d-1} \triangleleft \cdots \triangleleft H_{0}=G \text {. }
$$

We shall write $H \triangleleft^{d} G$. The least integer $d$ such that $H \triangleleft^{d} G$ is called the defect of $H$ in $G$. We define the terms $\gamma_{i}(G)$ of the lower central series of $G$ by $\gamma_{1}(G)=G$, 
$\gamma_{i}(G)=\left[G, \gamma_{i-1}(G)\right], i=2,3, \ldots$ A group $G$ is called nilpotent if $\gamma_{m}(G)=1$, for some integer $m$. If $c+1$ is the least value of $m$ satisfying this condition then $c$ is called the class of $G$.

\section{Statement and discussion of results}

We shall prove

Theorem 1. Let $R$ be a commutative ring with identity and let $\Omega$ be an infinite linearly ordered set. Whenever $G$ is a subgroup of $\operatorname{Sp}(\Omega, R)$ that contains $\operatorname{ESp}(\Omega, R)$ and $H$ is a subnormal subgroup of $G$ of defect $d$, then there exists an ideal $p$ of $R$ such that

$$
\operatorname{ESp}\left(\Omega,(4 \mathfrak{p})^{\mathcal{f ( d )}}\right) \leqq H \leqq S p^{\prime}(\Omega, \mathfrak{p})
$$

where $f(d)=\left(6^{d}-1\right) / 5$, for each integer $d \geqq 1$.

Notice that if $H$ is a normal subgroup of $S p(\Omega, R)$ then we can take $G=S p(\Omega, R)$ and $d=1$. If we assume that 2 is a unit in $R$ then we obtain the inclusions $E S p(\Omega, \mathfrak{p}) \leqq H \leqq S p^{\prime}(\Omega, \mathfrak{p})$. Conversely, if we assume that such inclusions hold for a subgroup $H$ of $S p(\Omega, R)$ then it follows from Lemma 2 that $H$ is normalised by $\operatorname{ESp}(\Omega, R)$. Thus, Theorem 1 reduces to the main theorem of $(6)$ when applied to normal subgroups of $S p(\Omega, R)$. In fact, our next result shows more clearly how Theorem 1 extends the work of (6).

Theorem 2. Let $R$ be a commutative ring in which 2 is a unit and let $H$ be a subgroup of $\operatorname{ESp}(\Omega, R)$. The following assertions are equivalent.

(i) $H$ is a subnormal subgroup of $\operatorname{ESp}(\Omega, R)$.

(ii) For some ideal $p$ of $R$ and some integer $m$

$$
\operatorname{ESp}\left(\Omega, \mathfrak{p}^{m}\right) \leqq H \leqq S p^{\prime}(\Omega, \mathfrak{p})
$$

We shall see that the ideal $p$ of Theorems 1 and 2 is $J(H)$, the level of $H$. We have remarked that the sandwiching

$$
\operatorname{ESp}(\Omega, J(H)) \leqq H \leqq S p^{\prime}(\Omega, J(H))
$$

of a subgroup $H$ of $E S p(\Omega, R)$ is a sufficient condition for the normality of $H$. However, the following example shows that, when 2 is not a unit in $R$, the sandwiching

$$
\operatorname{ESp}(\Omega, 4 J(H)) \leqq H \leqq S p^{\prime}(\Omega, J(H))
$$

of Theorem 1 is not in general a sufficient condition for the normality of $H$.

Example. If we let $N$ denote the set of natural numbers $\{1,2,3, \ldots\}$ and let $H$ be the subgroup of $\operatorname{ESp}(N, Z)$ generated by $\operatorname{ESp}(N, 4 Z)$ and $\left\{r_{i j}(n): i, j \in N, n \in Z\right\}$ then we see 
that $E S p(N, 4 Z) \leqq H \leqq S p^{\prime}(N, Z)$. However, $H$ is not a normal subgroup of $E S p(N, Z)$ since $t_{12}(1) \notin H$ while $t_{12}(1)=\left[r_{13}(1), s_{23}(1)\right] \in[H, E S p(N, Z)]$.

\section{Technical lemmas}

We begin with

Lemma 4. If $H$ is a subgroup of $S p(\Omega, R)$ that is normalised by $E S p(\Omega, \mathfrak{p})$, for some two-sided ideal $\mathfrak{p}$ of $R$ then $K(H)$ contains $y x$, for all generators $y$ of $J(H)$ and all $x \in \mathfrak{p}$.

Proof. Let $x \in \mathfrak{p}$. We must consider $y=A_{\mu \rho}$ or $y=A_{\mu \mu}-A_{\rho \rho}$, for some $\mu, \rho \in \Omega_{1}, \mu \neq \rho$ and $A \in H$. We examine only the case $\mu, \rho \in \Omega$ in detail; the other cases can be proved using similar arguments. First suppose $y=A_{\mu \rho}$. Pick $\lambda \in \Omega, \lambda \neq \rho, \mu$ and put $t=t_{\rho \lambda}(x)$. Then $\left[t, A^{-1}\right] \in \operatorname{ESp}(\Omega, R) \cap H, \quad$ by hypothesis and so for all $\psi \in \Omega_{1}, \quad \psi \neq \mu$, $\left[t, A^{-1}\right]_{\mu \psi} \in K(H)$ and $\left[t, A^{-1}\right]_{\mu \mu}-1 \in K(H)$, since elements of $\operatorname{ESp}(\Omega, R)$ differ from the identity matrix in only finitely many rows. But

$$
\left[t, A^{-1}\right]_{\mu \psi}=A_{\mu \rho} x A_{\lambda \psi}^{-1}-A_{\mu \lambda^{\prime}} x A_{\rho^{\prime} \psi}^{-1}, \quad \psi \neq \mu
$$

and

$$
\left[t, A^{-1}\right]_{\mu \mu}-1=A_{\mu \rho} x A_{\lambda \mu}^{-1}-A_{\mu \lambda^{\prime}} x A_{\rho^{\prime} \mu}^{-1}
$$

But

$$
A_{\mu \rho} x=\left(\left[t, A^{-1}\right]_{\mu \mu}-1\right) A_{\mu \lambda}+\sum_{\psi \neq \mu}\left[t, A^{-1}\right]_{\mu \psi} A_{\psi \lambda}
$$

and hence $A_{\mu \rho} x \in K(H)$.

It remains to show that $K(H)$ contains $\left(A_{\mu \mu}-A_{\rho \rho}\right) x$. If we examine $\left[t, A^{-1}\right]_{\rho \psi}$ and $\left[t, A^{-1}\right]_{\rho \rho}-1$, applying the same technique as above, we see that $A_{\rho \rho} x-x A_{\lambda \lambda} \in K(H)$ where $\lambda$ is chosen as above. Similarly we see that $K(H)$ contains $A_{\mu \mu} x-x A_{\lambda \lambda}$ and hence $K(H)$ contains $\left(A_{\mu \mu}-A_{\rho \rho}\right) x$.

Lemma 4 allows us to deduce

Corollary 1. If $H$ is a subgroup of $\operatorname{Sp}(\Omega, R)$ that is normalised by $\operatorname{ESp}(\Omega, \mathfrak{p})$, for some two-sided ideal $\mathrm{p}$ of $R$, then $J(H) \mathrm{p} \leqq K(H)$.

Next we prove

Lemma 5. Let $R$ be a commutative ring and let $H$ be a subgroup of $S p(\Omega, R)$ that is normalised by $\operatorname{ESp}(\Omega, \mathfrak{p})$, for some ideal $\mathfrak{p}$ of $R$. Let $A \in H \cap \operatorname{ESp}(\Omega, R)$ and let $x$ be any generator of $J(A)$. Let $u_{i} \in p, i=1, \ldots, 5$, let $y=4 u_{5} u_{4} u_{3} u_{2} u_{1} x$ and let $\lambda, \mu \in \Omega, \lambda \neq \mu$. $H$ contains $r_{\lambda \mu}(y), r_{\lambda \lambda}(y), s_{\lambda \mu}(y)$ and $s_{\lambda \lambda}(y)$. 
Proof. We may suppose that $x \neq 0$, for otherwise there is nothing to prove. We must consider $x=A_{\alpha \beta}$ or $x=A_{\alpha \alpha}-1$, for some $\alpha, \beta \in \Omega_{1}, \alpha \neq \beta$. We examine only the case $\alpha, \beta \in \Omega$ in detail; the other cases can be proved using similar arguments.

First suppose that $x=A_{\alpha \beta}$. Let $\phi \in \Omega, \phi \neq \lambda, \mu, \alpha, \beta$, be such that the $\phi$ th column and the $\phi^{\prime}$ th row of $A$ are trivial. (A row (column) is said to be trivial if it is equal to the corresponding row (column) of the identity matrix.) From Lemma 1 we see that

$$
\left[r_{\phi \beta}\left(-u_{2}\right),\left[t_{\phi \alpha}\left(u_{1}\right), A\right]\right]=r_{\phi \phi}\left(2 u_{2} u_{1} x\right)
$$

for any $u_{1}, u_{2} \in p$. Let $\psi \in \Omega, \psi \neq \phi, \lambda, \mu, \alpha, \beta$ be such that the $\psi^{\prime}$ th row and the $\psi$ th column of $A$ are trivial:

$$
\left[r_{\phi \phi}(y), t_{\psi \phi}(u)\right]=r_{\psi \phi}(-u y) r_{\psi \psi}\left(u^{2} y\right)
$$

shows that $H$ contains $r_{\psi \phi}\left(2 u_{3} u_{2} u_{1} x\right)$, since $r_{\psi \psi}\left(u^{2} y\right) \in H$ by (1). Finally the identities

$$
\begin{aligned}
& r_{\lambda \mu}\left(4 u_{5} u_{4} u_{3} u_{2} u_{1} x\right)=\left[r_{\psi \phi}\left(2 u_{3} u_{2} u_{1} x\right), t_{\mu \phi}\left(u_{4}\right), t_{\lambda \psi}\left(2 u_{5}\right)\right] \\
& r_{\lambda \lambda}\left(4 u_{5} u_{4} u_{3} u_{2} u_{1} x\right)=\left[r_{\psi \phi}\left(2 u_{3} u_{2} u_{1} x\right), t_{\lambda \phi}\left(u_{4}\right), t_{\lambda \psi}\left(u_{5}\right)\right] \\
& s_{\lambda \mu}\left(4 u_{5} u_{4} u_{3} u_{2} u_{1} x\right)=\left[s_{\phi \lambda}\left(2 u_{5}\right),\left[s_{\psi \mu}\left(-u_{4}\right), r_{\psi \phi}\left(2 u_{3} u_{2} u_{1} x\right)\right]\right] \\
& s_{\lambda \lambda}\left(4 u_{5} u_{4} u_{3} u_{2} u_{1} x\right)=\left[s_{\phi \lambda}\left(u_{5}\right),\left[s_{\psi \lambda}\left(-u_{4}\right), r_{\psi \phi}\left(2 u_{3} u_{2} u_{1} x\right)\right]\right]
\end{aligned}
$$

show that $H$ contains the required matrices when $x=A_{\alpha \beta}$.

Now suppose that $x=A_{\alpha \alpha}-1$. If we let $\phi$ be as above then Lemma 1 shows that

$$
r_{\phi \phi}\left(2 u_{2} u_{1} x\right)=\left[r_{\phi \alpha}\left(-u_{2}\right),\left[t_{\phi \alpha}\left(u_{1}\right), A\right]\right]
$$

The proof of this case is now completed using repeated applications of Lemma 1, as above.

We are now able to deduce

Corollary 2. If $R$ is a commutative ring and if $H$ is a subgroup of $S p(\Omega, R)$ that is normalised by $\operatorname{ESp}(\Omega, \mathfrak{p})$, for some ideal $\mathfrak{p}$ of $R$, then $H$ contains $\operatorname{ESp}\left(\Omega, 4 \mathfrak{p}^{6} J(H)\right)$.

\section{The proof of the theorems}

We begin with the proof of Theorem 1. Let $H, G, d$ and $f$ be as in the statement of the theorem. Whenever $\mathfrak{p} \leqq \mathfrak{q}, S p^{\prime}(\Omega, \mathfrak{p}) \leqq S p^{\prime}(\Omega, \mathfrak{q})$. Thus, since $X \in S p^{\prime}(\Omega, J(X))$, from the remarks following Lemma 3 , we see that $X \in S p^{\prime}(\Omega, J(H))$, for all $X \in H$. Hence $H \leqq S p^{\prime}(\Omega, J(H))$. It remains to prove the first inclusion and to do this we shall use an inductive argument. If $d=1$ then $H$ is normalised by $E S p(\Omega, R)$ so that $H$ contains

$$
\operatorname{ESp}\left(\Omega, 4 R^{6} J(H)\right)=\operatorname{ESp}(\Omega, 4 J(H))
$$


Now suppose that $E S p\left(\Omega,(4 J(K))^{f(k)}\right) \leqq K$, for all subgroups $K$ with subnormal chains of length $k<d$. If we put $J_{0}=J\left(H_{d-1}\right)$, where

$$
H=H_{d} \triangleleft H_{d-1} \triangleleft^{d-1} G,
$$

then we see that $H_{d-1}$ contains $\operatorname{ESp}\left(\Omega,\left(4 J_{0}\right)^{f(d-1)}\right)$. But $J=J(H) \leqq J_{0}$ so that $H$ contains

$$
\operatorname{ESp}\left(\Omega, 4(4 J)^{6 f(d-1)} J\right)=E S p\left(\Omega,(4 J)^{f(d)}\right)
$$

by Corollary 2 . We conclude, by the principle of induction, that whenever $H$ is as in the statement of Theorem $1, \operatorname{ESp}\left(\Omega,(4 \mathfrak{p})^{f(d)}\right) \leqq H$, where $\mathfrak{p}$ is the level of $H$. This, together with our initial remarks, completes the proof of Theorem 1.

We now prove Theorem 2. The proof of (i) implies (ii) follows from Theorem 1. Suppose therefore that (ii) holds. Since $\left(E S p(\Omega, R) \cap S p^{\prime}(\Omega, \mathfrak{p})\right) / E S p\left(\Omega, \mathfrak{p}^{m}\right)$ is nilpotent there is a series

$$
\operatorname{ESp}\left(\Omega, \mathfrak{p}^{m}\right) \leqq \gamma_{m} \leqq \cdots \leqq \gamma_{1}=\operatorname{ESp}(\Omega, R) \cap S p^{\prime}(\Omega, \mathfrak{p})
$$

where $\gamma_{i}=\gamma_{i}\left(E S p(\Omega, R) \cap S p^{\prime}(\Omega, p)\right), i=1, \ldots, m$. Hence

$$
H \leqq \gamma_{m} H \leqq \cdots \leqq \gamma_{1} H \leqq E S p(\Omega, R)
$$

is a normal series from $H$ to $\operatorname{ESp}(\Omega, R)$ of length $m+1$ and we conclude that $H$ is a subnormal subgroup of $\operatorname{ESp}(\Omega, R)$ of defect at most $m+1$.

\section{Acknowledgement}

The results of this paper form part of my doctoral thesis (1). I should like to thank my supervisor Dr. E. F. Robertson for the help and encouragement given to me while I was his student.

\section{REFERENCES}

(1) D. G. Arrell, Infinite dimensional linear and symplectic groups (Ph.D. thesis, University of St. Andrews, 1979).

(2) D. G. Arrell, The normal subgroup structure of the infinite general linear group; Proc. Edinburgh Math. Soc. 24 (1981), 197-202.

(3) H. Bass, Algebraic $K$-theory (Benjamin, New York-Amsterdam, 1968).

(4) J. Dieudonne, On the structure of the unitary groups, Trans. Amer. Math. Soc. 72 (1952), 367-385.

(5) J. Dieudonne, On the structure of the unitary groups, II, Amer. J. Math. 75 (1953), 665-678. 259.

(6) G. Maxwell, Infinite symplectic groups over rings, Comment. Math. Helv. 47 (1972), 254 
(7) E. F. Rober TSon; Some properties of $S p_{\Omega}(R)$, J. London Math. Soc. (2) 4 (1971), 65-78.

(8) E. SPIEGEL, On the structure of the infinite dimensional unitary group, Math. Annalen 172 (1967), 197-202.

(9). J. S. WiLson, The normal and subnormal structure of the general linear group, Proc. Cambridge Philos. Soc. 71 (1972), 163-177.

School of Mathematics ANd Computing

Leeds Polytechnic

LeEDS LS1 3HE 